\title{
Rotation Invariant Texture Classification Using Gabor Wavelets
}

\author{
Qingbo Yin ${ }^{1,2}$, Jong Nam Kim ${ }^{1}$, and Kwang-Seok Moon ${ }^{1}$ \\ ${ }^{1}$ Division of Electronic Computer and Telecommunication Engineering, Pukyong National \\ University, 599-1 Daeyeon-dong Nam-gu, Busan, 608-737, Korea \\ jongnam@pknu.ac.kr, ksmoon@pknu.ac.kr \\ ${ }^{2}$ College of Computer Science and Technology, Harbin Engineering University, P.R. China \\ yinqingbo@hrbeu.edu.cn
}

\begin{abstract}
This paper presents a new method for rotation invariant texture classification based on Gabor wavelets. The Gabor representation has been shown to be optimal in the sense of minimizing the joint two-dimensional uncertainty in space and frequency, and the Gabor wavelet can be used to decompose an image into multiple scales and multiple orientations. Two group features, i.e. the global feature vector and local feature matrix, can be constructed by the mean and variance of the Gabor filtered image. The global feature vector is rotation invariant, and the local feature matrix can be adjusted by a circular shift operation to rotation invariant so that all images have the same dominant direction. By the two group features, a discriminant can be found to classify the rotated images. In the primary experiments, comparatively high correct classification rates were obtained using a large sample sets with 1998 rotated images of 111 Brodazt texture classes.
\end{abstract}

Keywords: Texture Classification, Gabor Wavelet.

\section{Introduction}

Texture analysis is a fundamental issue in image analysis and computer vision, and has many potential applications, for example, in object recognition, biomedical image analysis and so on. Texture analysis has been an active research topic for more than three decades, but only a limited number of examples of successful exploration of texture exist. A major problem is that textures in the real world are often not uniform due to variations in orientation, scale, or other visual appearance.

Analysis of texture requires the identification of proper attributes or features that differentiate the textures for classification. There are numerous algorithms in the open literature for texture feature extraction and classification. The most common approaches to texture classification assume, either explicitly or implicitly, that all images are captured under the same orientation and the unknown samples to be classified are identical to the training samples with respect to spatial scale and orientation. However, it is unrealistic to control the environment to ensure a zero rotation angle.

In general, the approach towards developing rotation invariant techniques has been to modify successful non-rotation invariant techniques such as statistical methods, 
MRF (Markov random fields) and so on [1]. These traditional statistical approaches to texture analysis such as co-occurrence matrices, second order statistics, GaussMarkov random fields and local linear transforms, are restricted to the analysis of spatial interaction over relatively small neighborhoods on a single scale.

More recently, multi-resolution and multi-channel techniques have gained much attention for texture analysis, such as wavelet transform and Gabor filters. Based on filters with Gabor wavelet or other basis functions, the rotation invariant is realized by computing rotation invariant features from the filtered images or by converting rotation variant features to rotation invariant features. Porter and Canagarajah compared three mainstream paradigms: wavelets, GMRF, Gabor for rotation invariant classification [2]. The wavelet transform decomposes an image into only three orientations, i.e., horizontal, diagonal and vertical detail sub bands in the direction of $0^{\circ}, 45^{\circ}$ and $90^{\circ}$, respectively, apart from the approximation smooth sub-band. This limits the application of wavelet transform for rotation invariant texture analysis [3].

From two aspects of theory analysis and practices, it is true that Gabor filter has obvious advantages, compared with other methods. For feature-based approaches, rotation invariant is achieved by using anisotropic features. Gabor function can be appropriately considered as an orientation and scale tunable detector. The banks of Gabor filters are a group of wavelet, which one can capture the signal or feature at a specific frequency and a specific orientation. There are some approaches based on Gabor filters, which focus on the rotation invariant texture in [3-8], but most of them only work well on a small database and need a lot of samples for training. Haley and Manjunath used 13 classes of textures to obtain $96.4 \%$ correct classification, however only $80.4 \%$ on 109 classes of textures [4]. Tan used 15 classes of textures to obtain $89.3 \%$ correct classification [5]. Manthalkar and Biswas obtained 81.02\% correct classification on 60 classes of textures [6].

The motivation of this paper is to classify rotation invariant textures in a large texture database from the Brodatz album with 1998 rotated texture images derived from them by extracting global and local Gabor wavelet based features. The algorithm discussed in this paper is based on a feature space constructed from Gabor filter responses, in which each texture has the corresponding (unique) global feature and circular shift local feature. Then, a similarity measure, which combined the global and local features, is used to compare the unknown samples with the feature of known textures. The primary experiments have proven that the approach performs well in applications, and only requires one sample of each texture class at $0^{\circ}$ for training.

\section{Gabor Wavelet}

A two-dimensional Gabor function consists of a complex sinusoidal plane wave of some frequency and orientations, modulated by a two- dimensional Gaussian envelope. A 'canonical' Gabor filter $g(x, y)$ and its Fourier transform $G(u, v)$ can be written as: 


$$
\begin{gathered}
g(x, y)=\frac{1}{2 \pi \sigma_{x} \sigma_{y}} \exp \left[-\frac{1}{2}\left(\frac{x^{2}}{\sigma_{x}^{2}}+\frac{y^{2}}{\sigma_{y}^{2}}\right)\right] \cdot \exp (j 2 \pi W x) \\
G(u, v)=\exp \left\{-\frac{1}{2}\left[\frac{(u-W)^{2}}{\sigma_{u}^{2}}+\frac{v^{2}}{\sigma_{v}^{2}}\right]\right\}
\end{gathered}
$$

Where $\sigma_{u}=1 / 2 \pi \sigma_{x}$, and $\sigma_{v}=1 / 2 \pi \sigma_{y}$. Gabor functions form a complete but nonorthogonal basis set. Expanding a signal using this basis provides a localized frequency description. A class of self-similar functions, referred to as Gabor wavelets in the following discussion, is now considered. Let $g(x, y)$ be the mother Gabor wavelet, then this self-similar filter dictionary can be obtained by appropriate dilations and rotations of $g(x, y)$ through the generating function [9]:

$$
\begin{gathered}
g_{m n}(x, y)=a^{-m} g\left(x^{\prime}, y^{\prime}\right), a>1, m, n=\text { integer } \\
x^{\prime}=a^{-m}(x \cos \theta+y \sin \theta), y^{\prime}=a^{-m}(-x \sin \theta+y \cos \theta)
\end{gathered}
$$

Where $\theta=n \pi / K, k$ is the total number of orientations, $n$ is the orientation and $m$ is the scale of the Gabor wavelet, respectively. According to the scheme, the space frequency plane is covered nearly uniformly. The scale factor $a^{-m}$ in Eq.(3) ensures that the energy is independent of $m$.

$$
E_{m n}=\int_{-\infty-\infty}^{+\infty} \int_{-\infty}^{+\infty}\left|g_{m n}(x, y)\right|^{2} d x d y
$$

Let $U_{l}$ and $U_{h}$ represent the lower and upper center frequencies of interest. Let $K$ be the number of orientations and $S$ be the number of scales in the decomposition. Then, the design strategy is to ensure that half peak magnitude cross-section of the filter responses in the frequency spectrum touch each other. This results in the following formulas for computing the filter parameters $\sigma_{u}$ and $\sigma_{v}$ (and thus $\sigma_{x}$ and $\sigma_{y}$ ).

$$
\begin{gathered}
a=\left(U_{h} / U_{l}\right)^{1 / s-1} \\
\sigma_{u}=\frac{(a-1) U_{h}}{(a+1) \sqrt{2 \ln 2}} \\
\sigma_{v}=\tan \left(\frac{\pi}{2 k}\right)\left[U_{h}-2 \ln 2\left(\frac{\sigma_{u}^{2}}{U_{h}}\right)\right]\left[2 \ln 2-\frac{(2 \ln 2)^{2} \sigma_{u}^{2}}{U_{h}^{2}}\right]^{-1 / 2}
\end{gathered}
$$

Where $W=U_{h}, \theta=\pi / K$, and $m=0,1, \ldots, S-1$. 
Most Gabor filters have a slight response to the absolute intensity. This results in sensitivity to the background luminance level, which signifies a first order difference between regions. This can be avoided by adding a constant to make them zero mean. Because the Gabor filter is built in the Fourier domain, this same purpose (effect) is achieved by setting $\mathrm{G}(0,0)=0$.

\section{Feature Extractions and Classification}

\subsection{Texture Representation}

Given an image $I(x, y)$ of size $M * N$, its discrete Gabor wavelet transform is given by convolution:

$$
H_{m n}(x, y)=\sum_{p} \sum_{q} I(x-p, y-q) g_{m n}^{*}(p, q)
$$

Where $p, q$ are the filter mask size variables, $g_{m n}^{*}(p, q)$ is the complex conjugate of $g_{m n}$.

A set of Gabor wavelet of different scale and orientation is convolved with an image to estimate the magnitude of local frequency of the appropriate scale and orientation.

It is assumed that the texture regions are spatially homogeneous. So after applying Gabor filters on the image with orientation at different scale, the 'energy' content is obtained using:

$$
E(m, n)=\sum_{M} \sum_{N}\left|H_{m n}(x, y)\right|
$$

The mean $\mu_{m n}$ and standard deviation $\sigma_{m n}$ of the magnitude of the transform coefficients are used to construct two-group (local and global) features to represent the homogeneous textures.

$$
\mu_{m n}=\frac{E(m, n)}{M * N}, \sigma_{m n}=\frac{\sqrt{\sum_{x} \sum_{y}\left(\left|H_{m n}(x, y)\right|-\mu_{m n}\right)^{2}}}{M * N}
$$

If $\sigma_{m n}$ and $\mu_{m n}$ are combined to a vector $r\left(W_{m}, \theta_{n}\right)=\left(\mu_{m n}, \sigma_{m n}\right)$ as the unique feature at a certain orientation at a specific scale, the first group feature is denoted as the local feature:

$$
F G=\left(\begin{array}{ccc}
r\left(W_{0}, \theta_{0}\right) & r\left(W_{0}, \theta_{1}\right) & \cdots r\left(W_{0}, \theta_{K-1}\right) \\
r\left(W_{1}, \theta_{0}\right) & r\left(W_{1}, \theta_{1}\right) \cdots r\left(W_{1}, \theta_{K-1}\right) \\
\vdots & \vdots & \vdots \\
r\left(W_{S-1}, \theta_{0}\right) & r\left(W_{S-1}, \theta_{1}\right) \cdots r\left(W_{S-1}, \theta_{K-1}\right)
\end{array}\right)
$$


For convenience, typical matrix indexing is used in $F G, F G(1,1)=r\left(W_{0}, \theta_{0}\right)$ and $F G_{S K}=r\left(W_{S-1}, \theta_{K-1}\right)$. Another group of the texture features is obtained from sum of a certain frequency $W_{m}$ at all orientations:

$$
E_{m}=\sum_{n} r\left(W_{m}, \theta_{n}\right)
$$

Because the scheme of Gabor filter design have covered all space frequency plane nearly uniformly, a global rotation invariant feature vector can formed as $E=\left[E_{1}, E_{2}, \ldots E_{S}\right]$.

\subsection{Rotation Invariant Measurement}

In this paper, the anisotropic textures are emphasized as which we paid more attentions to them. Because a texture is perfectly homogeneous and isotropic, any texture descriptor would be rotation invariant. So, due to the anisotropic textures, a hypothesis is always correct in saying that there is a dominant pair of orientation and frequency in its Fourier spectrum, which means there is a $r\left(W_{m}, \theta_{n}\right)$ with highest energy.

Now, a column-wise circular shift of local feature matrix $F G$ can be defined as:

$$
F G^{k}=(F G(1: S, k: K) \quad F G(1: S, 1: k-1))
$$

This column-wise circular shift operation is used to rearrange local feature matrix $F G$ so that its dominant orientation can be in the first column of $F G^{\prime}=F G^{k}$.

Now, the direct distance metric can be defined between the query image $Q$ and a target image $T$ in the database as:

$$
\begin{gathered}
\left|r\left(W_{m}, \theta_{n}\right)\right|=\sqrt{\left(\mu_{m n}\right)^{2}+\left(\sigma_{m n}\right)^{2}}, r\left(W_{m}, \theta_{n}\right) \in G^{\prime} \\
d_{m n}(Q, T)=\left|r^{Q}\left(W_{m}, \theta_{n}\right)-r^{T}\left(W_{m}, \theta_{n}\right)\right|=\sqrt{\left(\mu_{m n}^{Q}-\mu_{m n}^{T}\right)^{2}+\left(\sigma_{m n}^{Q}-\sigma_{m n}^{T}\right)^{2}}
\end{gathered}
$$

Based on the local feature matrix $F G^{\prime}$ and global feature vector $E$, three differential factors can be defined as:

$$
d f_{E}=\frac{\sum_{m=1}^{S}\left(E_{m}^{Q}-E_{m}^{T}\right)^{2}}{\sum_{m=1}^{S}\left(E_{m}^{T}\right)^{2}}
$$




$$
\begin{gathered}
d f_{F G}=\frac{\sum_{m=1}^{S} \sum_{n=1}^{K}\left(d_{m n}\right)^{2}}{\sum_{m=1}^{S} \sum_{n=1}^{K}\left|r^{T}\left(W_{m}, \theta_{n}\right)\right|^{2}} \\
d f_{D}=\sum_{m=1}^{S}\left(E_{m}^{Q}-E_{m}^{T}\right)^{2}+\sum_{m=1}^{S} \sum_{n=1}^{K}\left(d_{m n}\right)^{2}
\end{gathered}
$$

Actually, in this paper, the texture similarity measurements are accomplished by defining the differentia measurement:

$$
d f=\min \left(d f_{E} * d f_{F G} * d f_{D}\right)
$$

This similarity measurement is the rotation invariant, and emphasis that variety ratio of global and local 'energy' between the query image $Q$ and a target image $T$ is smallest if $Q$ is the same as or very similar to $T$.

\section{Experiments}

In order to test the efficiency of the proposed method for rotation invariant texture analysis, experiments are carried out on a texture database from the Brodatz texture album, which is comprised of 111 texture images of size $512 \times 512$. Each center portion of size $256 \times 256$ from the respective texture image of size $512 \times 512$ is used in the training phase. In the same way, the samples for testing can be obtained

\begin{tabular}{|c|c|c|c|c|c|c|c|c|}
\hline \multicolumn{2}{|c|}{ Parameters } & \multirow{2}{*}{$\begin{array}{c}\text { Correct } \\
\text { classifica- } \\
\text { tion rate }\end{array}$} & \multicolumn{2}{|c|}{ Parameters } & \multirow{2}{*}{$\begin{array}{l}\text { Correct } \\
\text { classifica- } \\
\text { tion rate }\end{array}$} & \multicolumn{2}{|c|}{ Parameters } & \multirow{2}{*}{$\begin{array}{c}\text { Correct } \\
\text { classifica- } \\
\text { tion rate }\end{array}$} \\
\hline Scale & $\begin{array}{c}\text { Orienta- } \\
\text { tion }\end{array}$ & & Scale & $\begin{array}{l}\text { Orienta- } \\
\text { tion }\end{array}$ & & Scale & $\begin{array}{c}\text { Orienta- } \\
\text { tion }\end{array}$ & \\
\hline 3 & 4 & $85.0 \%$ & 4 & \multirow{2}{*}{$\begin{array}{l}4 \\
5\end{array}$} & $91.3 \%$ & 5 & 4 & $93.6 \%$ \\
\hline 3 & 5 & $87.7 \%$ & 4 & & $92.7 \%$ & 5 & 5 & $92.9 \%$ \\
\hline 3 & 6 & $88.3 \%$ & 4 & 6 & $94.8 \%$ & 5 & 6 & $95.1 \%$ \\
\hline 3 & 7 & $88.6 \%$ & 4 & 7 & $92.8 \%$ & 5 & 7 & $92.3 \%$ \\
\hline 3 & 8 & $90.2 \%$ & 4 & 8 & $95.3 \%$ & 5 & 8 & $95.2 \%$ \\
\hline 3 & 9 & $89.5 \%$ & 4 & 9 & $92.9 \%$ & 5 & 9 & $91.7 \%$ \\
\hline 3 & 10 & $91.3 \%$ & 4 & 10 & $95.1 \%$ & 5 & 10 & $95.1 \%$ \\
\hline 3 & 11 & $92.0 \%$ & 4 & 11 & $92.8 \%$ & 5 & 11 & $92.7 \%$ \\
\hline 3 & 12 & $90.1 \%$ & 4 & 12 & $96.0 \%$ & 5 & 12 & $95.3 \%$ \\
\hline \multicolumn{2}{|c|}{$\begin{array}{l}\text { Mean with } \\
\text { scale=3 }\end{array}$} & $89.2 \%$ & \multicolumn{2}{|c|}{$\begin{array}{l}\text { Mean with } \\
\text { scale }=4\end{array}$} & $93.7 \%$ & \multicolumn{2}{|c|}{$\begin{array}{l}\text { Mean with } \\
\text { scale }=5\end{array}$} & $93.8 \%$ \\
\hline
\end{tabular}

Table 1. Results of rotated texture classification using the proposed approach with various scale and orientation values 
but rotated in steps of $10^{\circ}$ up to $180^{\circ}$. Texture classification is done with a total of 1998 rotated texture images $(111 \times 18=1998)$ for various combinations of scale and orientation values of Gabor decomposition (scale is from 3 up to 5; orientation from 4 up to 12). The center frequencies for Gabor filters are 0.05-0.4 and separated by one octave-frequency bandwidth.

The results obtained for various combinations of scale and orientation values of Gabor decomposition are given in Table 1. From Table 1, it can be shown that the lowest mean correct classification rate of $85.0 \%$ is obtained at scale $=3$ and orientation=4 with 28 features ( 24 features of local feature matrix $G$ plus 4 features of global feature vector $E$ ), and the highest mean correct classification rate of $96.0 \%$ is obtained at scale $=4$ and orientation $=12$ with 100 features $(96$ features of local feature matrix $G$ plus 4 features of global feature vector $E$ ). The next highest mean correct classification rate of $95.3 \%$ is at scale $=5$ and orientation $=12$ with 125 features (120 features of local feature matrix $G$ plus 5 features of global feature vector $E$ ), and at scale $=4$ and orientation $=8$ with 68 features ( 64 features of local feature matrix $G$ plus 4 features of global feature vector $E$ ). And it is showed that the correct classification rates increase approximately with scales and orientations increasing. But, when scale is larger than 4, there is no vast difference between the mean classification rate obtained for different combination of scale and orientation values of Gabor decomposition. In practice, the best combination can be decided by taking into account the tradeoff (compromise) of the number of features and the minimum correct classification rate.

\section{Conclusions}

A rotation invariant texture classification scheme using two group features (global feature vector and local feature matrix) based on the Gabor wavelet is developed for a reasonably large (111 classes) texture databases. Two group features, i.e. the global feature vector and local feature matrix, can be constructed by the mean and variance of the Gabor filtered image. Global feature vector is rotation invariant, and local feature matrix can be adjusted by a circular shift operation to rotation invariant so that all images have the same dominant direction. By the two group features, a discriminant can be found to classify rotated images. The primary experiments have proven that the proposed approach is effective for rotation invariant texture classification.

There are many application areas such as automated inspection, large image database handling, remote sensing and medical image processing. Further research should include its robustness to image noise, and scale invariant texture classification.

\section{Acknowledgements}

This work was supported by The Regional Research Centers Program (Research Center for Logistics Information Technology), granted by the Korean Ministry of Education \& Human Resources Development. 


\section{References}

1. Jianguo Zhang, Tieniu Tan.: Brief review of invariant texture analysis methods. Pattern Recognition, Vol. 35(3), (2002) 735-747.

2. Porter, R., Canagarajah, N.: Robust rotation-invariant texture classification: Wavelet, Gabor filter and GMRF based schemes. IEE Proceedings of Image Signal Processing 144 (3), 1997.

3. Arivazhagan, S., Ganesan L., and Padam Priyal, S.: Texture classification using Gabor wavelets based rotation invariant features. Pattern Recognition Letters, Vol.27(16), (2006) 1976-1982

4. Haley, G.M., Manjunath, B.S.: Rotation-invariant texture classification using a complete space-frequency model. IEEE Transactions on Image Processing 8 (2), (1999) 169-255.

5. Tan, T.N.: Rotation invariant texture features and their use in automatic script identification. IEEE Transactions on Pattern Analysis and Machine Intelligence 20, (1998) 751-756.

6. Manthalkar, R., Biswas, P.K. and Chatterji, B.N.: Rotation invariant texture classification using even symmetric Gabor filters. Pattern Recognition Letter, Vol.24(12), (2003) 20612068

7. Jianguo Zhang and Tieniu Tan.: New texture signatures and their use in rotation invariant texture classification. Proceedings of Texture 2002 (The 2nd international workshop on texture analysis and synthesis with ECCV 2002)

8. Jianguo Zhang, Tieniu Tan, Li Ma.: Invariant texture segmentation via circular gabor filter. Proceedings of the 16th IAPR International Conference on Pattern Recognition (ICPR), Vol II, (2002) 901-904.

9. Manjunath, B.S., Ma, W.Y.: Texture features for browsing and retrieval of image data. IEEE Transactions on Pattern Analysis and Machine Intelligence, Vol.18(8), (1996) $837-842$ 